\title{
Pretreatment with myo-inositol in non polycystic ovary syndrome patients undergoing multiple follicular stimulation for IVF: a pilot study
}

Franco Lisi ${ }^{1 *}$, Piero Carfagna ${ }^{1}$, Mario Montanino Oliva ${ }^{1}$, Rocco Rago ${ }^{1}$, Rosella Lisi ${ }^{1}$, Roberta Poverini ${ }^{1}$, Claudio Manna ${ }^{2}$, Elena Vaquero ${ }^{3}$, Donatella Caserta ${ }^{4}$, Valeria Raparelli ${ }^{5}$, Roberto Marci ${ }^{6}$ and Massimo Moscarini ${ }^{4}$

\begin{abstract}
Background: Aim of this pilot study is to examine the effects of myo-inositol administration on ovarian response and oocytes and embryos quality in non PolyCystic Ovary Syndrome (PCOS) patients undergoing multiple follicular stimulation and in vitro insemination by conventional in vitro fertilization or by intracytoplasmic sperm injection.

Methods: One hundred non-PCOS women aged $<40$ years and with basal FSH $<10 \mathrm{mUI} / \mathrm{ml}$ were down-regulated with triptorelin acetate from the mid-luteal phase for 2 weeks, before starting the stimulation protocol for oocytes recovery. All patients received $\mathrm{FFSH}$, at a starting dose of $150 \mathrm{IU}$ for 6 days. The dose was subsequently adjusted according to individual response. Group $B(n=50)$ received myo-inositol and folic acid for 3 months before the stimulation period and then during the stimulation itself. Group A ( $n-50)$ received only folic acid as additional treatment in the 3 months before and through treatment.

Results: Total length of the stimulation was similar between the two groups. Nevertheless, total amount of gonadotropins used to reach follicular maturation was found significantly lower in group B. In addition, the number of oocytes retrieved was significantly reduced in the group pretreated with myo-inositol. Clinical pregnancy and implantation rate were not significantly different in the two groups.

Conclusions: Our findings suggest that the addition of myo-inositol to folic acid in non PCOS-patients undergoing multiple follicular stimulation for in-vitro fertilization may reduce the numbers of mature oocytes and the dosage of rFSH whilst maintaining clinical pregnancy rate. Further, a trend in favor of increased incidence of implantation in the group pretreated with myo-inositol was apparent in this study. Further investigations are warranted to clarify this pharmacological approach, and the benefit it may hold for patients.
\end{abstract}

Keywords: Myo-inositol, Inositol, Follicle, Stimulation, IVF, ICSI, Oocytes, Embryos

\section{Background}

Myo-inositol is an isomer of a C6 sugar alcohol that belongs to the vitamin B complex group [1]. Some studies suggested that myo-inositol could play an important role in cellular morphogenesis and cytogenesis, in the synthesis of lipids, in the creation of cell membranes and in cell growth [2,3]. It is also a precursor of phospholipids, which are responsible for the generation of important intracellular signals in mammalian oocytes and in the resumption of meiotic maturation [4-6]. The

\footnotetext{
* Correspondence: franco.lisi01@gmail.com

${ }^{1}$ Center for Reproductive Medicine Research, Clinica Villa Mafalda, Rome, Italy Full list of author information is available at the end of the article
}

presence of myo-inositol in human body fluids and its effect on the in vitro maturation of oocytes in rats have led some authors to state that myo-inositol concentration in follicular fluid is significantly higher in follicles containing good quality oocytes than in follicles containing poor quality oocytes [7].

Myo-inositol also regulates, via signal transduction pathways, the secretion of some exocrine glands such as pancreas and other organs, including the ovaries. In the oocytes these intracellular pathways are involved in the release of cortical granules, in the inhibition of polyspermy, in the completion of meiosis and in the activation of the cell cycle that subsequently results in

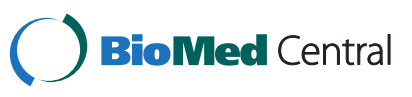


embryonic development [8]. It has been hypothesized that intrafollicular myo-inositol concentration and oocyte quality might be connected because inositol phospholipids (of which myo-inositol is a precursor) are held responsible for important intracellular signals essential for oocyte development, and because myo-inositol itself seems to improve oocytes in vitro maturation $[8,9]$.

Recently, the role of myo-inositol has powerfully emerged in the pathogenesis of polycystic ovary syndrome (PCOS), in particular linked with insulin resistance. In fact, some of the actions of insulin are mediated by putative inositol-containing phosphoglycan (IPG) mediators, also known as putative insulin mediators or second messengers. These mediators are generated by hydrolysis of glycosylphosphatidylinositol lipids and/or proteinated species located in the outer leaflet of the cell membrane. Two different IPG have been identified: (i) the D-chiro-IPG mediator, which activates pyruvate dehydrogenase phosphatase, and (ii) the MYO-IPG which inhibits cyclic AMP-dependent protein kinase [10,11]. A positive role of myo-inositol in insulin-resistant women with PCOS could depend on defects in the insulin IPGmediated signaling pathway, that seems to be primarily implicated in the pathogenesis of insulin resistance in this clinical setting [12,13]. Accordingly, myo-inositol has been classified as an insulin sensitizing agent and it is commonly used in PCOS treatment [14-16]. By rescuing the ovarian response to endogenous gonadotropins, myo-inositol reduces hyperandrogenemia and reestablishes menstrual cyclicity and ovulation, increasing the chance of a spontaneous pregnancy $[17,18]$. Its use in human is safe and only the highest dose (12 g/day) induced mild gastrointestinal side effects such as nausea, flatus and diarrhea [19].

The aim of this paper was to illustrate the progress recently made in the use of myo-inositol in fertility treatment and in particular to discuss its effects on ovarian response and oocyte quality in non-PCOS patients undergoing multiple follicular stimulations and in vitro inseminations by conventional in vitro fertilization (IVF) or by intracytoplasmic sperm injection (ICSI).

\section{Methods}

This prospective, randomized, open-label, multicenter pilot study compared patients treated with $400 \mu \mathrm{g}$ of folic acid for the 3 months before and during rFSH administration, following the long protocol (Group A, $\mathrm{n}=50$ ) with patients that received a daily dose of $4,000 \mathrm{mg}$ of myo-inositol into two administrations/day in addition to $400 \mu \mathrm{g}$ of folic acid for the 3 months before and during $\mathrm{rFSH}$ administration, following the long protocol (Group B, $n=50$ ). The trial adhered to the Helsinki Declaration and the protocol was approved by the Institutional Review Boards. All patients signed a written informed consent before entering the study. Inclusion criteria were age $<40$ years old and basal FSH level $<10$ $\mathrm{mUI} / \mathrm{ml}$. Patients presenting diagnostic criteria for PCOS [14] or other concomitant endocrine and metabolic diseases such as hypothyroidism, hyperthyroidism, diabetes mellitus, androgen-secreting tumors, adrenal hyperplasia, Cushing's syndrome, hyper-prolactinemia, and all patients that underwent hormonal treatment in the previous 3 months were excluded from the study. Day $2 \mathrm{FSH}, \mathrm{LH}, 17 \beta$-estradiol (E2) and prolactin (PRL) levels were measured in the previous 6 months. Patients' BMI was between 18 and 28 and obese women (BMI greater than $30 \mathrm{~kg} / \mathrm{m}^{2}$ ) were excluded from the study: Six patients of the initial cohort had a BMI greater than $30 \mathrm{~kg} / \mathrm{m} 2$ (6/198; 3\%). All patients began treatment during a set period -January 2011 to January 2012 ("treatment run") - and were allocated to the treatment groups using block randomization in a computer generated sequence: computer generated numbers 1-10000 into two columns - Column A was rFSH and folic acid using the 'even integers'; Column B was rFSH and folic acid with addition of myo-inositol using odd integers (Figure 1). Allocation to group A or group B was decided on the day of first consultation and was not known to doctors who performed the monitoring of follicular development, changes in the amount of daily International Units of $\mathrm{rFSH}$, egg retrieval, in vitro fertilization, embryo transfer and decided luteal support.

Patients enrolled in the study were recommended ICSI or IVF after evaluation of their partner's semen. Patients were down-regulated with triptorelin acetate $(0.1 \mathrm{mg}$, SC; Ferring, Italy) from the mid-luteal phase (day 21 of the previous cycle) for 2 weeks, before starting the stimulation protocol for oocyte recovery. In all patients, ovarian suppression was confirmed by ultrasound scan (absence of ovarian activity, ovarian cyst formation and endometrial proliferation) and serum dosage of E2 levels $(\leq 40 \mathrm{pg} / \mathrm{ml})$ before starting exogenous gonadotropins administration. If ovarian suppression was not met, down-regulation was extended for a further week. Group A received folic acid at a dose of $400 \mu \mathrm{g}$ per day for 3 months before and during treatment with gonadotropins and received $\mathrm{rFSH}$ (Gonal F, Merck) at a starting dose of $150 \mathrm{IU}$ for 6 days. The dose was subsequently adjusted according to individual response. Group B received the same gonadotropins treatment and the stimulation was preceded by the administration of 2,000 mg of myo-inositol twice a day and $400 \mu \mathrm{g}$ per day of folic acid for 3 months before starting follicular stimulation and continued during the stimulation itself. When conventional criteria for human chorionic gonadotropin (hCG) administration were met (at least three follicles with a mean diameter $>17 \mathrm{~mm}$ ), recombinant human hCG, $(250$ mg SC; Ovitrelle, Merck 


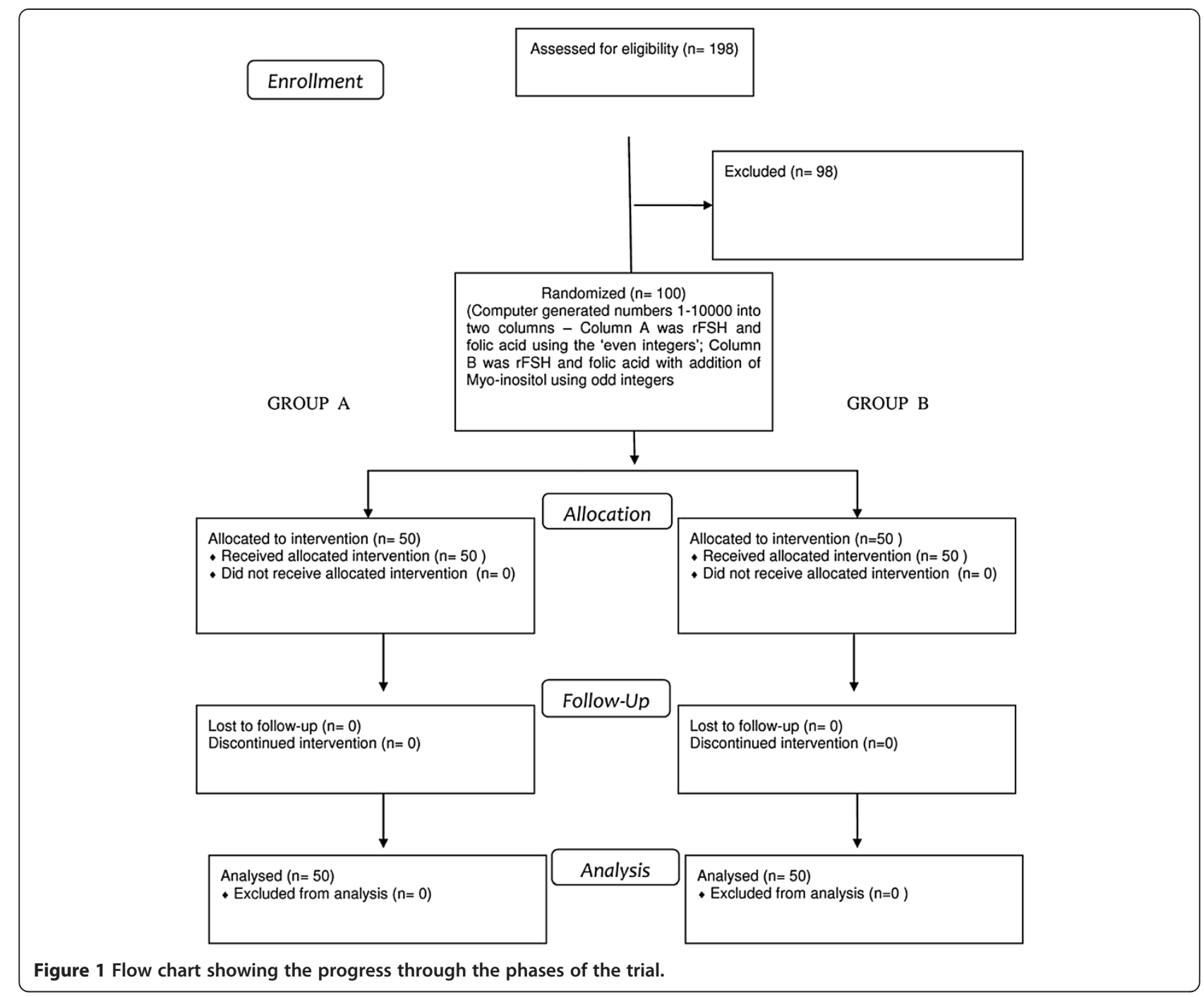

Serono) was given (at least 24 hours after the last $\mathrm{rFSH}$ administration) to induce final oocyte maturation. Transvaginal oocyte retrieval was scheduled 35-37 hours after the trigger injection. IVF or ICSI, depending on semen parameters (data not shown), were then performed. Oocytes retrieval, IVF or ICSI and embryos transfer were carried out according to our usual clinical practice [20]. Embryo quality was assessed morphologically, 2 days after fertilization using a grading system [21]. Grade 1 and 2 embryos have no or very few fragments in the cytoplasm with equal size blastomeres and therefore are considered the best embryos. Grade 3 and 4 embryos have significant or severe fragmentation; little cytoplasmic fragmentation with blastomeres of distinctively unequal size [21]. The luteal phase was supported by $800 \mathrm{mg} /$ day transvaginal-micronized progesterone (Progeffik, Effik Italia S.p.A, Milan, Italy) and treatment was continued until either a serum pregnancy test result was negative or an embryonic heart beat was sonographically confirmed. Serum hCG level was measured 14 days after oocyte retrieval. A slight and transitory increase in $\beta$-hCG level was defined as a biochemical pregnancy. A gestational sac with fetal heartbeat movement seen on transvaginal ultrasound scan 4 weeks after embryo transfer, confirmed clinical pregnancy.

\section{Statistical analysis}

The number of treated patients and controls was computed with respect to a two-tailed Student $t$ test for independent groups, considering a (i) difference in gonadotropins dosage required to reach follicular maturation to be detected between patients and controls $|\delta| \geq 15 \%$, (ii) type I error probability $\alpha=0.05$ and power $1-\beta=0.72$; this resulted in $n=50$ for group. Sample size calculation was performed using the software nQuery Advisor, version 5.0 (Statistical Solutions, Saugus, Massachusetts). Analysis was performed according to an 
intention to treat principle. Student's $t$-test for independent samples was used to evaluate statistical differences between groups for continuous variables. Comparisons between proportions were conducted using the Pearson's chi-square test.

Data are presented as mean \pm SD. Probability values $<0.05$ were regarded as statistically significant. All calculations were made with the computer programme STATISTICA 7 (StatSoft, Tulsa, OK, USA).

\section{Results and discussion}

Patients' age was similar in the two groups as well as BMI, FSH, LH and E2 basal levels. After the downregulation treatment, $\mathrm{LH}$ level was significantly higher in patients pre-treated with myo-inositol [Group A: $1.6 \pm 0.9 \mathrm{mUI} / \mathrm{ml}$; Group B: $2.7 \pm 1.1 \mathrm{mUI} / \mathrm{ml}, \mathrm{p}<0.01]$, while basal FSH and basal E2 were similar (Table 1). The total length of the stimulation was similar between the two groups [Group A: $11.7 \pm 1.8$ days; Group B: $11.8 \pm 1.5$ days; $\mathrm{p}=0.64]$. The total amount of gonadotropins used to reach follicular maturation was significantly reduced in group B [Group A: 2,479 \pm 979 IU; Group B: 2,084 \pm 648 IU; p <0.05]. E2 peak level on the day of hCG administration was found to be lower, but not statistically significant, in group A [Group A: $1.312 \pm 629$; Group B $1.516 \pm 942 \mathrm{pg} / \mathrm{ml}, \mathrm{p}=0.12$ ] and the number of oocytes per patient retrieved resulted

Table 1 Baseline and stimulation characteristics of patients in group without (Group A) and with (Group B) pretreatment with Myo-inositol

\begin{tabular}{|c|c|c|c|}
\hline & $\frac{\text { No myo-inositol }}{\text { Group A }}$ & $\frac{\text { Yes myo-inositol }}{\text { Group B }}$ & p \\
\hline & $\mathrm{N}=50$ & $\mathrm{~N}=50$ & \\
\hline No. of patients & 50 & 50 & \\
\hline Age (year) & $33.3 \pm 2.8$ & $34.4 \pm 3.4$ & 0.09 \\
\hline$B M l$ & $22.9 \pm 3.3$ & $22.7 \pm 2.6$ & 0.88 \\
\hline FSH (m/U/ml), basal & $7.3 \pm 1.5$ & $7.2 \pm 2$ & 0.35 \\
\hline LH (m/U/ml), basal & $4.7 \pm 2$ & $4.9 \pm 2$ & 0.87 \\
\hline $\begin{array}{l}\text { 17ß-estradiol (E2) (pg/ml), } \\
\text { basal }\end{array}$ & $47.2 \pm 17.8$ & $43.6 \pm 12.7$ & 0.20 \\
\hline $\begin{array}{l}\text { FSH }(\mathrm{m} / \mathrm{U} / \mathrm{ml}) \text {, down } \\
\text { regulation }\end{array}$ & $4.7 \pm 1.5$ & $4.6 \pm 2.2$ & 0.87 \\
\hline $\begin{array}{l}L H(m / U / m l), \text { down } \\
\text { regulation }\end{array}$ & $1.6 \pm 0.9$ & $2.7 \pm 1.1$ & $<0.01$ \\
\hline $\begin{array}{l}\text { 17ß-estradiol (E2) }(\mathrm{pg} / \mathrm{ml}) \text {, } \\
\text { down regulation }\end{array}$ & $26.4 \pm 20$ & $24.4 \pm 14.5$ & 0.31 \\
\hline rFSH treatment days & $11.7 \pm 1.8$ & $11.8 \pm 1.5$ & 0.64 \\
\hline Total rFSH dose (IU) & $2,479 \pm 979$ & $2,084 \pm 648$ & $<0.05$ \\
\hline E2 $(\mathrm{pg} / \mathrm{ml})$, day of $h C G$ & $1,312 \pm 629$ & $1,516 \pm 640$ & 0.12 \\
\hline Total length of stimulation & $11.7 \pm 1.8$ & $11.8 \pm 1.5$ & 0.64 \\
\hline
\end{tabular}

significantly higher in group A [Group A: 7.6 \pm 3.8 per patient (Tot. 380); Group B: 5,9 $\pm 2,4$ oocytes per patient (Tot. 297), $\mathrm{p}<0.01$ ] (Table 2). In addition the number (mean $\pm \mathrm{SD}$ ) of Metaphase II eggs, number of inseminated eggs, number of $2 \mathrm{PN}$ oocytes and number of embryos were significantly higher in group A compared with group B (Table 2). Fertilization rate, cleavage rate, percentage of grade I and II embryos, number of patients receiving embryos and number of embryos received were similar in both groups (Table 2).

The number of clinical pregnancies was also similar [Group A: $12 / 47$ (25,5\%); Group B: 14/47 (29,8\%), $\mathrm{p}=0.52$ ]. Finally, even the implantation rate (number of gestational sac with fetal heartbeat/total number of embryos transferred) was similar in the two groups of patients [Group A: 13,3\%; Group B: 18,7\%, p =0.08] (Table 2).

PCOS affects $5 \%-10 \%$ of women in reproductive age, and is the most common cause of infertility due to anovulation. Insulin resistance is common in PCOS women, regardless of their body mass index. The importance of insulin resistance in PCOS is also suggested by the fact that insulin-sensitizing compounds have been proposed as putative treatments to solve hyperinsulinemiainduced dysfunction of ovarian response to endogenous gonadotropins. Rescuing ovarian response to endogenous gonadotropins reduces hyperandrogenemia and reestablishes menstrual cyclicity and ovulation, increasing the chance of a spontaneous pregnancy. Among insulinsensitizing compounds, myo-inositol has been shown to be able to restore spontaneous ovarian activity, and consequently fertility, in most patients with PCOS $[18,22,23]$. Myo-inositol may be a useful tool in the treatment of PCOS patients undergoing ovulation induction for its insulin-sensitizing activity. Myo-inositol has also been proposed as an adjuvant in multiple follicular stimulation for IVF in patients known to suffer from PCOS : the pretreatment with myo-inositol and folic acid was shown to reduce germinal vesicles and degenerated oocytes at ovum pick-up, without compromising the total number of retrieved oocytes $[8,24]$.

However, the intent of this study was to assess the role of myo-inositol in cellular morphogenesis and cytogenesis. At present not many data are available regarding the action and effects of myo-inositol in non-PCOS women in childbearing age, in spontaneous ovulation and in stimulation cycles. Increasing evidence supports the physiological and therapeutic role of myo-inositol in human reproduction and in particularly in oogenesis playing an important role in cell morphogenesis and cytogenesis, lipid synthesis, structure of cell membranes and cell growth $[2,3]$. Some studies have shown that myo-inositol is incorporated into phosphoinositides and inositol phosphates in rabbit embryos [25] and can 
Table 2 Study outcomes in the two treatment groups

\begin{tabular}{|c|c|c|c|}
\hline & $\frac{\frac{\text { No myo-inositol }}{\text { Group A }}}{\mathrm{N}=50}$ & $\begin{array}{c}\frac{\text { Yes myo-inositol }}{\text { Group B }} \\
N=50\end{array}$ & $p$ \\
\hline No. oocytes retrieved/patient & $7.6 \pm 3.8$ & $5.9 \pm 2.4$ & $<0.01$ \\
\hline \multicolumn{4}{|l|}{ Mean $\pm S D$} \\
\hline No. metaphase II/patient & $6.3 \pm 2.9$ & $4.8 \pm 2.2$ & $<0.05$ \\
\hline \multicolumn{4}{|l|}{ Mean $\pm S D$} \\
\hline No. inseminated eggs/patient & $6 \pm 2.7$ & $4.8 \pm 2.2(240)$ & $<0.05$ \\
\hline \multicolumn{4}{|l|}{ Mean $\pm S D$} \\
\hline No. 2PN oocytes/patient & $4.3 \pm 2.3$ & $3.3 \pm 1.8 / 163$ & $<0.01$ \\
\hline \multicolumn{4}{|l|}{ Mean $\pm S D$} \\
\hline Fertilization rate (2PN/inseminated oocytes) & $70.8 \pm 20.4$ & $68.4 \pm 19.2$ & 0.7 \\
\hline \multicolumn{4}{|l|}{ (\%) $\pm S D$} \\
\hline No. Embryos/patient & $3.58 \pm 2.1$ & $2.5 \pm 1.1$ & $<0.001$ \\
\hline \multicolumn{4}{|l|}{ Mean $\pm S D$} \\
\hline Cleavage rate (embryos/2PN oocytes) & $82.2 \pm 29.9$ & $84.5 \pm 28.7$ & 0.7 \\
\hline \multicolumn{4}{|l|}{ (\%) $\pm S D$} \\
\hline No. embryos Grade I and II : number & $173(96.6 \%)$ & $117(93.6 \%)$ & 0.2 \\
\hline \multicolumn{4}{|l|}{ and percentage of total } \\
\hline No. of patients receiving & 47 (94\%) & $47(94 \%)$ & 1 \\
\hline \multicolumn{4}{|l|}{ embryos (\%) } \\
\hline No. of embryos transferred per & $2.4 \pm 1$ & $2.2 \pm 0.8$ & 0.39 \\
\hline \multicolumn{4}{|l|}{ starting patients Mean $\pm S D$} \\
\hline No. Clinical pregnancies & $12 / 47(25.5 \%)$ & 14/47 (29.8\%) & 0.52 \\
\hline No. of fetal hearts & $16(13.3 \%)$ & $21(18.7 \%)$ & 0.08 \\
\hline (implantation rate) & & & \\
\hline
\end{tabular}

enhance bovine blastocyst development from in vitro culture with medium supplemented with myo-inositol [26]. Results from these studies support the notion that myo-inositol serves as a precursor for the synthesis of phosphoinositides. This constitutes the phosphatidylinositol (PtdIns) signal transduction system known to be involved in the regulation of diverse cellular functions including cell proliferation [27]. During ovulation induction for an IVF cycle two important parameters need to be monitored: E2 concentration and follicles size/number. An increase in these two factors has been correlated with a higher level of myo-inositol in the follicular fluid [7]. In 1992 Chiu and Tam [28] demonstrated that serum myo-inositol could be a trophic factor responsible for promoting in vitro development of preimplantation embryos.

Myo-inositol is an important element of the follicular microenvironment that plays a crucial role in oocyte maturation. In fact, in Assisted Reproduction, supplementation of myo-inositol is positively related to oocyte meiotic progression of germinal vesicles in rats, increasing the intracellular calcium oscillations $[4,28,29]$. In patients treated with exogenous gonadotropins plus myo-inositol there was a significant reduction in the number of oocytes retrieved and in the number of follicles recruited. For this reason we can assume that this approach could be adopted to reduce the risk of hyperstimulation. Overall, these results provide a further support to the hypothesis that myo-inositol may promote the meiotic maturation acting on intracellular signal transduction in calcium pathways [29-31]. A complete meiotic maturation requires intracellular changes associated with both nuclear and cytoplasmic components [30]. At present, although most of the morphological and biochemical changes during maturation are well documented, a complete identification of specific factors that directs these changes is lacking [32]. The fertilizability of oocytes, their ability to initiate embryo splitting, and the subsequent preimplantation development are now considered a fundamental part of a proper assessment of cytoplasmic maturation [33]. It is interesting to highlight how LH level during down regulation 
was higher in the group pretreated with myo-inositol [Group A: $1.6 \pm 0,9 \mathrm{mIU} / \mathrm{ml}$; Group B: $2.7 \pm 1.1 \mathrm{mIU} / \mathrm{ml}$, $\mathrm{p}<0.01]$. Although the meaning of this finding is not clear, we can speculate that the increased rate of circulating LH is responsible for the higher level of E2 on the day of HCG administration (although not significant in our group of patients) and for the lower number of follicles recruited, suggesting that it may be a cofactor for better oocytes and embryos quality [34].

\section{Conclusion}

The addition of myo-inositol seems to reduce gonadotropin dosage and the number of MII oocytes retrieved in non-PCOS patients pretreated with myo-inositol for 3 months. However, this study is underpowered to evaluate IVF outcomes like implantation and clinical pregnancy with the mechanism of improved oocyte competence. Therefore a subsequent adequately powered RCT is underway.

\section{Competing interests}

The authors declare that they have no competing interests.

\begin{abstract}
Author details
${ }^{1}$ Center for Reproductive Medicine Research, Clinica Villa Mafalda, Rome, Italy. ${ }^{2}$ GENESIS Center for Reproductive Medicine, Rome, Italy. ${ }^{3}$ Department of Surgery, Tor Vergata - University of Rome, Rome, Italy. ${ }^{4}$ Department of Women Health and Territorial Medicine, Sapienza - University of Rome, Sant'Andrea Hospital, Rome, Italy. ${ }^{5}$ Department of Internal Medicine and Medical Specialties, Sapienza - University of Rome, Rome, Italy. ${ }^{6}$ Department of Biomedical Sciences and Advanced Therapies, Section of Obstetrics and Gynecology, University Hospital of Ferrara, Ferrara, Italy.
\end{abstract}

\section{Authors' contributions}

All the authors participated in designing the study, patients' enrollment, analysis of results and preparation of manuscript. FL, PC, MMO, RR, RL, RP, $C M, E V, D C, R M, M M$ participated in designing, recruiting and treating patients, in the analysis of results and the preparation of the manuscript. VR participated in the analysis of data and in the preparation of manuscript. All authors read and approved the final version of the manuscript.

Received: 14 April 2012 Accepted: 9 July 2012

Published: 23 July 2012

\section{References}

1. Kane MT: The effects of water-soluble vitamins on the expansion of rabbit blastocysts in vitro. J Exp Zool 1988, 245:220-223.

2. Berridge MJ: Inositol lipids and cell proliferation. Biochim Biophys Acta 1987, 907:33-45.

3. Downes CP: Twenty-fifth Colworth medal lecture. The cellular functions of myo-inositol. Biochem Soc Trans 1989, 17:259-268.

4. Akiba S, Sato T: Cellular function of calcium-independent phospholipase A2. Biol Pharm Bull 2004, 27:1174-1178.

5. Aouameur R, Da Cal S, Bissonnette P, Coady MJ, Lapointe JY: SMIT2 mediates all myo-inositol uptake in apical membranes of rat small intestine. Am J Physiol Gastrointest Liver Physiol 2007, 293:G1300-1307.

6. Smith SD, Mikkelsen A, Lindenberg S: Development of human oocytes matured in vitro for 28 or 36 hours. Fertil Steril 2000, 73:541-544.

7. Chiu TT, Rogers MS, Law EL, Briton-Jones CM, Cheung LP, Haines CJ: Follicular fluid and serum concentrations of myo-inositol in patients undergoing IVF: relationship with oocyte quality. Hum Reprod 2002, 17:1591-1596

8. Papaleo E, Unfer V, Baillargeon JP, Chiu TT: Contribution of myo-inositol to reproduction. Eur J Obstet Gynecol Reprod Biol 2009, 147:120-123.
9. Papaleo E, Unfer V, Baillargeon JP, Fusi F, Occhi F, De Santis L: myo-inosito may improve oocyte quality in intracytoplasmic sperm injection cycles. A prospective, controlled, randomized trial. Fertil Steril 2009, 91:1750-1754.

10. Larner J: Mediators of postreceptor action of insulin. Am J Med 1983, 74:38-51.

11. Saltiel AR: Second messengers of insulin action. Diabetes Care 1990, 13:244-256.

12. Nestler JE, Jakubowicz DJ, Reamer P, Gunn RD, Allan G: Ovulatory and metabolic effects of D-chiro-inositol in the polycystic ovary syndrome. N Engl J Med 1999, 340:1314-1320.

13. Cheang Kl, Baillargeon JP, Essah PA, Ostlund RE Jr, Apridonize T, Islam L, Nestler JE: Insulin-stimulated release of D-chiro-inositol-containing inositolphosphoglycan mediator correlates with insulin sensitivity in women with polycystic ovary syndrome. Metabolism 2008, 57:1390-1397.

14. Azziz R: PCOS: a diagnostic challenge. Reprod Biomed Online 2004, 8:644-648.

15. Baillargeon JP, luorno MJ, Nestler JE: Insulin sensitizers for polycystic ovary syndrome. Clin Obstet Gynecol 2003, 46:325-340.

16. Baillargeon JP, Diamanti-Kandarakis E, Ostlund RE Jr, Apridonidze T, luorno MJ, Nestler JE: Altered D-chiro-inositol urinary clearance in women with polycystic ovary syndrome. Diabetes Care 2006, 29:300-305.

17. Iuorno MJ, Jakubowicz DJ, Baillargeon JP, Dillon P, Gunn RD, Allan G, Nestler JE: Effects of d-chiro-inositol in lean women with the polycystic ovary syndrome. Endocr Pract 2002, 8:417-423.

18. Unfer V, Carlomagno G, Dante G, Facchinetti F: Effects of myo-inositol in women with PCOS: a systematic review of randomized controlled trials. Gynecol Endocrinol 2012, 28(7):509-515.

19. Carlomagno G, Unfer V: Inositol safety: clinical evidences. Eur Rev Med Pharmacol Sci, 15:931-936.

20. Fishel S, Lisi F, Rinaldi L, Green S, Hunter A, Dowell K, Thornton S: Systematic examination of immobilizing spermatozoa before intracytoplasmic sperm injection in the human. Hum Reprod 1995, 10:497-500.

21. Veeck LL: Atlas of the Human Oocyte and early conceptus. Baltimore: Williams \& Wilkins; 1986.

22. Baillargeon JP, Nestler JE, Ostlund RE, Apridonidze T, Diamanti-Kandarakis E: Greek hyperinsulinemic women, with or without polycystic ovary syndrome, display altered inositols metabolism. Hum Reprod 2008, 23:1439-1446.

23. Costantino D, Minozzi G, Minozzi E, Guaraldi C: Metabolic and hormonal effects of myo-inositol in women with polycystic ovary syndrome: a double-blind trial. Eur Rev Med Pharmacol Sci 2009, 13:105-110.

24. Ciotta L, Stracquadanio M, Pagano I, Carbonaro A, Palumbo M, Gulino F: Effects of inositol on oocyte quality in patients affected with polycystic ovary syndrome. Minerva Ginecol 2010, 62:525-531.

25. Fahy MM, Kane MT: Incorporation of [3 $\mathrm{H}$ ] inositol into phosphoinositides and inositol phosphates by rabbit blastocysts. Mol Reprod Dev 1993, 34:391-395.

26. Holm P, Booth PJ, Schmidt MH, Greve T, Callesen H: High bovine blastocyst development in a static in vitro production system using SOFaa medium supplemented with sodium citrate and myo-inositol with or without serum-proteins. Theriogenology 1999, 52:683-700.

27. Berridge MJ, Irvine RF: Inositol phosphate and cell signalling. Nature 1989, 306:197-205.

28. Chiu TT, Tam PP: A correlation of the outcome of clinical in vitro fertilization with the inositol content and embryotrophic properties of human serum. J Assist Reprod Genet 1992, 9:524-530.

29. Groenen PM, Merkus HM, Sweep FC, Wevers RA, Janssen FS, SteegersTheunissen RP: Kinetics of myo-inositol loading in women of reproductive age. Ann Clin Biochem 2003, 40:79-85.

30. Jedrusik A, Ajduk A, Pomorski P, Maleszewski M: Mouse oocytes fertilised by ICSI during in vitro maturation retain the ability to be activated after refertilisation in metaphase II and can generate Ca2+ oscillations. BMC Dev Biol 2007, 7:72.

31. Chiu TT, Rogers MS, Briton-Jones C, Haines C: Effects of myo-inositol on the in-vitro maturation and subsequent development of mouse oocytes. Hum Reprod 2003, 18:408-416.

32. Kurokawa M, Fissore RA: ICSI-generated mouse zygotes exhibit altered calcium oscillations, inositol 1,4,5-trisphosphate receptor-1 downregulation, and embryo development. Mol Hum Reprod 2003, 9:523-533. 
33. Fulka J Jr, First NL, Moor RM: Nuclear and cytoplasmic determinants involved in the regulation of mammalian oocyte maturation. Mol Hum Reprod 1998, 4:41-49.

34. Lisi F, Rinaldi L, Fishel S, Lisi R, Pepe GP, Picconeri MG, Campbell A: Use of recombinant $\mathrm{LH}$ in a group of unselected IVF patients. Reprod Biomed Online 2002, 2(5):104-108.

doi:10.1186/1477-7827-10-52

Cite this article as: Lisi et al:: Pretreatment with myo-inositol in non polycystic ovary syndrome patients undergoing multiple follicular stimulation for IVF: a pilot study. Reproductive Biology and Endocrinology 2012 10:52.

\section{Submit your next manuscript to BioMed Central and take full advantage of:}

- Convenient online submission

- Thorough peer review

- No space constraints or color figure charges

- Immediate publication on acceptance

- Inclusion in PubMed, CAS, Scopus and Google Scholar

- Research which is freely available for redistribution 